\title{
Sex and gender analysis in knowledge translation interventions: challenges and solutions
}

Amédé Gogovor ${ }^{1,2,3,4}$, Tatyana Mollayeva ${ }^{5,6}$, Cole Etherington ${ }^{7}$, Angela Colantonio ${ }^{5,6}$, France Légaré ${ }^{1,2,3,4^{*}}$ and on behalf of the GIKT Group

\begin{abstract}
Sex and gender considerations are understood as essential components of knowledge translation in the design, implementation and reporting of interventions. Integrating sex and gender ensures more relevant evidence for translating into the real world. Canada offers specific funding opportunities for knowledge translation projects that integrate sex and gender. This Commentary reflects on the challenges and solutions for integrating sex and gender encountered in six funded knowledge translation projects. In 2018, six research teams funded by the Canadian Institutes of Health Research's Institute of Gender and Health met in Ottawa to discuss these challenges and solutions. Eighteen participants, including researchers, healthcare professionals, trainees and members of the Institute of Gender and Health, were divided into two groups. Two authors conducted qualitative coding and thematic analysis of the material discussed. Six themes emerged, namely Consensus building, Guidance, Design and outcomes effectiveness, Searches and recruitment, Data access and collection, and Intersection with other determinants of health. Solutions included educating stakeholders on the use of sex and gender concepts, triangulating perspectives of researchers and end-users, and participating in organisations and committees to influence policies and practices. Unresolved challenges included difficulty integrating sex and gender considerations with principles of patient-oriented research, a lack of validated measurement tools for gender, and a paucity of experts in intersectionality. We discuss our findings in the light of observations of similar initiatives elsewhere to inform the further progress of integrating sex and gender into the knowledge translation of health services research findings.
\end{abstract}

Keywords: Gender, sex, healthcare, knowledge translation, research design, intersectionality

\section{Introduction}

For almost two decades, the Canadian Institutes of Health Research (CIHR) have been leaders in funding knowledge translation (KT) research, known elsewhere as implementation science. Coined by CIHR, KT is

\footnotetext{
* Correspondence: france.legare@fmed.ulaval.ca

${ }^{1}$ Vitam - Centre de recherche en santé durable, Pavillon Landry-Poulin, 2525, Chemin de la Canardière, Québec G1J OA4, Canada

${ }^{2}$ Tier 1 Canada Research Chair in Shared Decision Making and Knowledge Translation, Université Laval, Quebec, Canada

Full list of author information is available at the end of the article
}

defined as a dynamic and iterative process that includes synthesis, dissemination, exchange and ethically sound application of knowledge to improve the health of Canadians, provide more effective health services and products and strengthen the health care system [1]. This application of research knowledge can involve patients, clinicians, public health officials, health service managers and policy-makers $[2,3]$. KT interventions facilitate the uptake of research evidence into practice and/or policy, i.e. they are interventions that make use of research results [3]. KT interventions can include patient and 
clinician education programmes, evidence-based guidelines, policy-making, promotion of self-management or continuous quality improvement programmes.

However, it is now becoming clear that KT must take sex and gender into consideration, as these are critical equity issues for intervention uptake in areas such as decision-making and communication as well as for stakeholder engagement, needs and interests [4]. A KT strategy may function differently and has different impacts depending on the sex and gender identity of researchers, participants and end-users as well as on the gender relations among them. Theories and frameworks operate differently within and across sexes and genders. There is growing evidence too that applying a sex and gender perspective enhances the accuracy and relevance of findings. Moreover, if sex and gender are not considered, KT programmes can reproduce or even increase existing inequities $[4,5]$.

CIHR has been a leading institution in advancing sex and gender considerations in health research. Starting in 2013, the CIHR Institute of Gender and Health (IGH) initiated a strategic plan with a central focus on sex, gender and health to advance the integration of sex and gender considerations throughout all phases of the health research cycle [6]. For the purposes of this paper, we define sex and gender based on the CIHR definitions, as follows: sex refers to a set of biological attributes in humans and animals while gender refers to socially constructed roles, behaviours, expressions and identities of girls, women, boys, men and gender-diverse people [7]. Sex and gender interact and affect different aspects of social roles, relations, norms and identity [8-10]. White women and black women, for example, may share determining factors for health based on sex but other determining factors, such as race and ethnicity or class, may differ substantially [11]. Intersectionality is an approach to understanding and analysing how these different factors operate and influence each other in the organisation of power in a given society $[12,13]$. The intersectional lens is used in many studies that address sex and gender, including three out of the six KT projects we discuss [14-16].

We aimed to reflect on challenges and solutions found in integrating sex- and gender-based analysis (SGBA) into six KT interventions.

\section{The funded projects}

Six research teams were funded by the CIHR IGH to (1) generate evidence about whether applying SGBA to KT interventions involving human participants improves effectiveness; (2) contribute to a broader knowledge base on how to integrate sex and gender into KT interventions; and (3) facilitate the consideration and development of gender-transformative approaches (i.e. approaches that promote gender equity) in KT interventions $[6,17,18]$. The call echoed several international recommendations, including the Madrid Statement, urging $\mathrm{WHO}$ and the member states to include sexdisaggregated information in their data collection systems and develop gender-sensitive indicators to achieve the highest standard of health and support effective health policy $[19,20]$. These six ongoing projects (Box 1) are taking place in the context of teaching hospitals, community-based health and social centres, workplaces, professional organisations, and KT developers.

\section{Conduct of the meeting and synthesis of the discussions}

Eighteen participants (15 women and 3 men; 8 Frenchspeaking) including 9 researchers, 3 healthcare professionals, 4 trainees, 1 CIHR IGH staff member, and Dr. Cara Tannenbaum, Scientific Director of the CIHR IGH (cihr-irsc.gc.ca/e/12735.html), attended the meeting (Additional file 1 for agenda).

Prior to the meeting, team leaders were asked to identify three challenges they had met within the course of their project for which strategies were found and three challenges for which solutions had not yet been found. These lists of challenges with and without solutions were shared among teams ahead of the meeting along with recent relevant publications to give each team member time to consider ideas and prepare suggestions. Participants were divided into two discussion groups and, after the discussion, reported back to the larger group. Subsequently, each team revised or updated their pre-meeting texts based on the discussions. Afterwards, two authors (AG, TM) conducted a thematic analysis of the revised pre-meeting texts, the summaries reported back to the larger group, and notes taken by the team members during the discussions [21]. The two authors independently conducted inductive initial coding of the texts with iterative discussions for consensus throughout the process. Themes were reviewed by other members of the teams, including team members who were not present at the meeting (Additional file 2).

Verbal consent was obtained from the participants regarding further use of the output of the meeting. We received a waiver from the Centre intégré universitaire de santé et de services sociaux de la Capitale-Nationale Ethics Board.

\section{Challenges of integrating sex and gender into KT}

For some challenges, teams found solutions that fully resolved the problem. However, for others, there were issues for which none of the teams felt they had found adequate solutions. Challenges, solutions and unresolved challenges were organised into six themes and are 
presented following the phases of a KT intervention, namely Consensus building, Guidance, Design and outcomes effectiveness, Searches and recruitment, Data access and collection, and Intersection with other determinants of health.

\section{Consensus building}

Due to the interdisciplinary nature of sex and gender research as well as the importance of stakeholder involvement in KT, research teams could become quite large and unwieldy, with widely varying perspectives and levels of engagement. The teams found it difficult uniting around a common vision and a common language on sex and gender. For example, there was disagreement within research teams on defining sex and gender concepts (e.g. in the CPD team), on whether to treat sex and gender as non-binary variables and on whether there is enough evidence to include sex and gender considerations in clinical guidelines (e.g. in the TBI team).

Solutions: Better communication, i.e. sharing of material and a development of a consensus on terms and concepts before the implementation process begins, regular team meetings, monthly newsletters, and regular working sessions.

Unresolved: For the CPD team, developing a research process that (1) combines the world of sex and gender experts with that of KT experts, (2) uses a patientoriented research lens and (3) is committed to patient engagement, is a complex and as yet unresolved challenge. Another challenge still unresolved was successfully communicating the complexity and novelty of the topic of sex and gender to research partners. Another unresolved issue was research partners who do not engage and how to take them into account in the knowledge translation process.

\section{Guidance}

Teams found there was a lack of guidance regarding integrating concepts of sex and gender into models, frameworks or guidelines. For example, the OR team had difficulty integrating sex and gender considerations into the Theoretical Domains Framework (TDF), and the TBI team found a lack of theories to explain sex and gender influences in TBI.

Solutions: Regarding incorporating gender into the TDF, the OR team sought feedback from multiple coinvestigators with expertise in sex- and gender-based analysis. The team proposed that the TDF could be used as a coding structure only and that, rather than posing the traditional TDF questions, questions could incorporate the project context and be more free-flowing in style. The TBI team believed that, for the moment, the best approach was less direct, i.e. to capture sex and gender effects in TBI through the unique voices and narratives of women and men with TBI, their significant others, and clinicians.

\section{Design and effectiveness outcomes Study designs}

The methodological and ethical relevance of using study designs with control groups in studies initiated at the behest of or in partnership with sex or gender subgroups was questioned. Is it ethically sound or relevant to reproduce the intervention with another subgroup not mobilised in the same way by the same question? Another challenge is taking gender into consideration (as opposed to sex) in studies with small cohorts without losing statistical power and, finally, how to integrate gender into data analysis, for example, in studies on how firefighters' work or pregnant women's work affects their health (environmental/occupational health team).

The CPD team, who conducted a controlled trial, found limited evidence for adding sex- and gender-based content to their experimental CPD training on diabetes and depression. It also struggled with managing the new experimental training: it required more time than the control training that excluded it and risked diluting core health-related content. The control also risked reaffirming existing sex or gender biases in the original training. The same team, for example, noted that the intervention group noticed and appreciated sex and gender notions while the control group noticed (and complained about) the absence of sex and gender notions.

Solutions: Present more social factors in the control training. The CPD team added a qualitative dimension to the intervention in the form of an interactive component (following the theoretical component) including a brainstorming activity and a panel discussion on stigmatisation related to depression and/or diabetes, medication, and gender.

\section{Other design issues}

If investigators are not aware of their own biases or of power differentials, design methods risk re-naturalising or reaffirming sex or gender biases. There may be a power disequilibrium between researchers and partners or between partner A and partner B, resulting in some voices being louder than others in the KT process. As the environmental/occupational health team asked, are methods such as Delphi, for example (concept definition based on a consensus approach), valid in the presence of social groups that do not have the same power and voice strength?

Solutions: Identify methods that will produce valid and authentic data from excluded or less empowered populations. For example, the occupational/environmental health team advised 'don't mix men and women in the same focus group if you suspect a risk of power 
imbalance'. Give strength to the voices of the most excluded in dissemination initiatives.

\section{Effectiveness outcomes}

Identifying outcomes for evaluating community-based KT interventions that integrate sex and gender was a challenge for all teams. Which criteria should be used to determine its success or effectiveness? Effectiveness, as evaluated qualitatively by researchers versus by endusers or stakeholders, can be different, depending on their understanding or experience of sex and gender, and can complicate the dissemination of research results. In addition, the scientific community and academic journals are conservative about evaluation models and often closed to experimental ones.

Solution: Instead of using the controlled trial study design, the occupational/environmental health team conducted retrospective analyses, triangulated the perspectives of researchers and users, and focused on process and intermediate indicators by describing different interventions (case studies) rather than seeking to compare interventions that cannot be compared. Involve stakeholders in the co-construction and validation process, then triangulate views when interpreting and disseminating results. Findings should be interpreted and disseminated with a consciousness of sex and gender bias or stereotypes. For example, as one team noted, if injury risk factors are different for women and men, do not presume women are weaker. Therefore, it is important to carry out strategic and innovative initiatives to share knowledge with a broader audience, such as being on journal editorial boards, being peer reviewers, influencing journal ratings by selecting the best ones in regard to sex/gender considerations, and refusing to publish in journals that do not accept papers with innovative methods or topics.

\section{Searches and recruitment Searches}

Adding sex and gender to search terms in certain KT domains can unearth a huge and unmanageable volume and variety of returns. The substance use team, whose systematic review questions were multi-component (with four substances and three levels of intervention), had over 22,000 returns on their search.

Solutions: The substance use team narrowed the research question, modelled searches based on key papers and developed an SGBA categorisation for the prioritisation of papers.

\section{Recruitment}

It can be challenging to recruit participants who identify themselves as gender diverse to capture a complete spectrum of sex and gender data, risking their exclusion from interventions that could benefit them. For example, the TBI team were unable to recruit anyone who selfidentified as gender diverse.

Solutions: Connect with relevant organisations and develop trusting relationships with them. For example, the TBI team and the occupational/environmental health team contacted organisations with ties to the LGBTQ+ (Lesbian, gay, bisexual, transgender, queer, and other diverse options) community and created partnerships with them.

\section{Data access and data collection Usable data}

Baseline data, common definitions of terms, and socalled cleansed data (where incorrect, incomplete, improperly formatted, or duplicated data are amended or removed) are required metrics against which implemented changes can be measured. Context-specific data in a usable format (i.e. disaggregated) was not always available for these teams, for example, usable data relating to environmental and occupational health, on depression, and on diabetes. When data are presented by sex or gender, often, little attention is paid to explanatory hypotheses and mechanisms. Furthermore, in the literature, the terms "sex" and "gender" are frequently used interchangeably. The substance use team, who were conducting a systematic review, found that the term 'gender" is particularly misunderstood, often being interpreted as gender identity, as sex, or as referring to women only.

Solutions: Participation in organisations and publishing to influence policy and practice, giving lectures and conferences to relevant organisations. For example, the occupational/environmental health team founded the International Ergonomics Association Technical Committee on Gender and Work to lobby for more sex- and gender-sensitive investigations and interventions, organised and edited a special issue of the journal Applied Ergonomics dealing with gender issues in ergonomics, and gave lectures in universities and labour organisations to explain why sex- and gender-sensitive analysis contributes to better interventions.

\section{Data collection}

Teams struggled with a lack of understanding of the meaning of sex and gender among participants, regardless of whether they were patients, caregivers or clinicians. This lack of understanding could result in participants being alienated or offended by questions on sex and gender. The TBI team, for example, found that participants who were asked directly about sex and gender, especially those with experience of TBI, often confused the terms, conflated them with sexuality, or 
declined to answer the questions. In addition, some were unwilling to be educated on the topic.

Solutions: Although the TBI team used CIHR definitions to explain the terms, they explained that TBI frequently affects socially marginalised populations and found that, rather than asking direct questions, it is through asking about how patients' lives had been impacted by their injuries and hearing their stories that they come to understand their "roles, behaviors, and expectations" and gain a deeper understanding of participants' sex and gender constructs. The occupational/ environmental health team suggested using fact sheets with short, illustrative and shocking examples of how sex or gender bias can influence research results and practice and getting to know the point of view of stakeholders and other researchers before trying to educate them.

\section{The intersection of sex and gender with other determinants of health}

The intersectional team was confronted with the issue of how to ensure sex and gender are not diluted when considering their intersection with the multiple other determinants of health and the exclusion of other important determinants such as Indigeneity.

Solutions: The intersectional team found that the methods for striking a balanced representation of sex and gender considerations were capacity-building, a consensus process with all representative stakeholders, including in-person and online discussions, and finally voting on which frameworks and tools to use.

Unresolved: Paucity of experts in intersectionality, difficulty in operationalisation of intersectionality, and lack of researchers' experience in $\mathrm{KT}$.

\section{Discussion}

We reported the discussions of a meeting that was held in November 2018 in Ottawa to examine challenges CIHR-funded team members have faced and to share key strategies. Scientific methods to study sex and gender in health research are still evolving, with limited agreement between and within stakeholder groups on knowledge and education needs on the concept of sex and gender.

Although some of the challenges are relevant to sexand gender-sensitive research per se, others are specific to KT. These challenges are related to educating (sometimes unwilling) researchers and participants about sex and gender, understanding of terms, the unwieldy nature of large integrated KT projects that involve many disciplines and stakeholders, and integrating constructs of sex and gender into educational material for healthcare professionals and patients in the presence of limited scientific evidence on the topic.
The lack of guidance for integrating sex and gender into KT interventions made it difficult for these researchers to operationalise the construct, as did the confusion or disagreements over the terms sex and gender [22]. The concept of gender is particularly complex and was a major source of confusion [23]. While sex and gender are now recognised as non-binary variables and researchers are urged to move beyond binary conceptualisation of sex/gender $[22,24]$, with no methodological advice or evidencebased recommendations to guide them, it is still challenging for researchers to grapple with sex- and gender-based analysis in KT.

The challenges can occur at every stage and involve researchers (e.g. lack of theoretical guidance), patient experts and other stakeholders (e.g. disagreement on terms) or participants (e.g. recruitment). Different study designs had different challenges - for example, challenges to do with control interventions were relevant to trials, challenges with literature searches were especially relevant to a systematic review and challenges to do with power imbalances could particularly affect a Delphi consensus study.

The teams reported challenges pertaining to difficulties in recruiting gender-diverse participants and in assessing or collecting disaggregated data. These difficulties are compounded by the use of inconsistent terminology in the literature, difficulty in applying the concepts, a failure to come to terms with the impact of

Box 1 Settings and goals of the six Institute of Gender and Health-funded knowledge translation research projects (further details in Table 1)

Team 1, based in Ontario, Canada, is investigating the impact of gender on the effectiveness of teamwork in the operating room with clinicians in urban academic hospitals ('the OR team').

Team 2, based in Ontario, Canada, is documenting concepts and ideas for developing and testing educational materials on sex and gender for traumatic brain injury (TBI) care ('the TBI team').

Team 3, is performing a systematic review on evidence on sex and gender factors affecting substance use (alcohol, cannabis, nicotine and opioids) and is using the evidence in three pilot interventions on substance use in Manitoba, Nunavut and Saskatchewan, Canada ('the substance use team').

Team 4 seeks to model a sex- and gender-conscious participatory action approach in occupational health and environmental health interventions by unions and other community groups in the Amazon and in Quebec, Canada ('the occupational/environmental health team')

Team 5 aims to increase sex- and gender-sensitive clinical behaviours and attitudes through continuing professional development (CPD) activities in French-speaking urban and rural communities in Quebec, New Brunswick and Ontario, Canada ('the CPD team').

Team 6, a pan-Canadian team, aims to develop and evaluate intersectional approaches to KT frameworks and associated tools with KT intervention developers working with older adults ('the intersectional team'). 


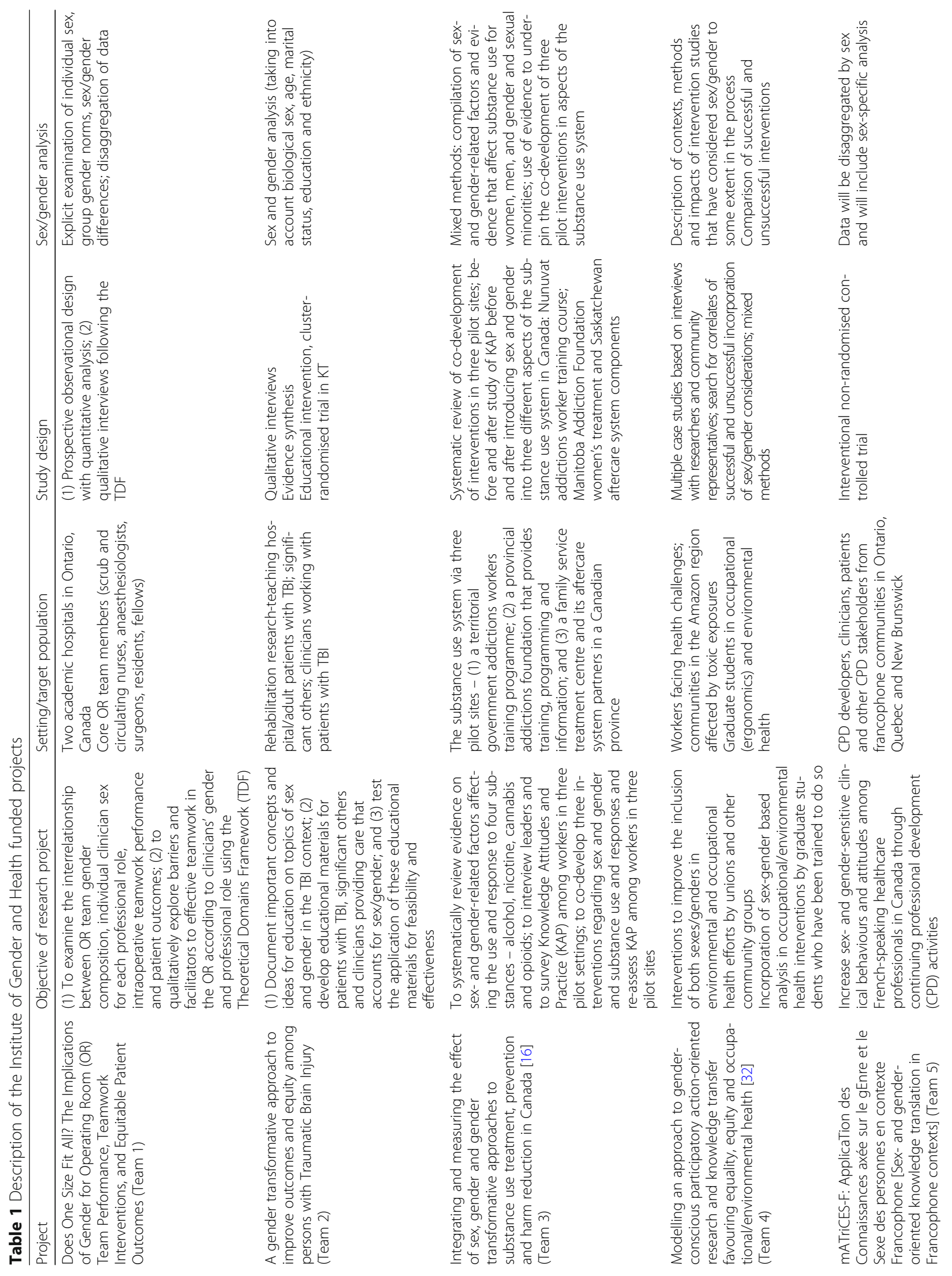




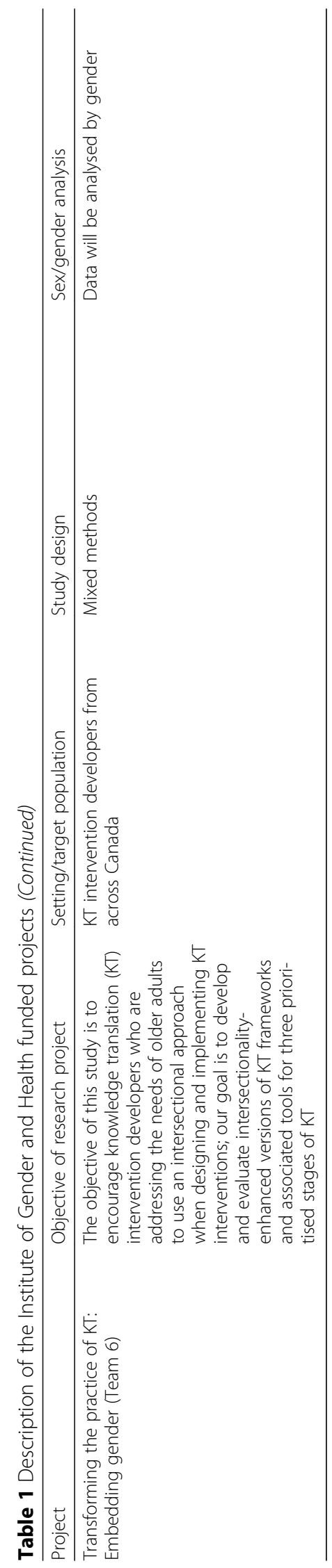


sex and gender on research design and outcomes, and challenges with data collection and datasets. These findings have been confirmed in a review of literature on sex- and gender-based analysis [25]. While progress has been made in this respect in some research fields, such social science health research [22, 26], implementation literature reveals that attention to sex and gender has not yet infiltrated research methods in the field of KT [4]. The strategies proposed by the teams may begin to fill this gap.

Another challenge was the difficulty in operationalisation of intersectionality. Various international bodies, including the WHO, have recognised the importance of intersectional research $[8,27]$. Recent calls for research strategies for both higher and low- and middle-income countries (LMICs) recommend sex and gender analysis as an entry point into a deeper intersectional analysis [27, 28]. Yet methods for integrating intersectionality into research and policy are still in their very early stages of development.

The problems of effectiveness outcomes found by the teams are interesting to compare with those found by ADVANCE teams (similar initiatives in the United States) and Athena SWAN teams (in the United Kingdom). The contribution of ADVANCE to increasing inclusivity has been difficult to separate from overall institutional and academic pushes for inclusivity; additionally, their projects include small numbers of individuals and multiple interventions, which makes it difficult to isolate change [11]. The Athena SWAN project also found it difficult to directly link the effects of policy changes with their initiatives [29].

Finally, these difficulties or challenges experienced by the teams, both those with solutions and those that remain unresolved, share one striking element when considered as a whole - they are all struggling to deal with complexity. The difficulty of operationalising intersectionality in $\mathrm{KT}$ studies is related to the complexity of considering so many determining factors together. The difficulty of theorising or developing models or guidelines on sex and gender considerations is related to the fact that they are fluid and deeply complex notions. The difficulty of using controlled trial design in integrating sex and gender into KT relates to the complexity of assessing their components and determining any meaningful outcome. The difficulties of managing large multidisciplinary $\mathrm{KT}$ teams that include sex and gender experts as well as patients and other stakeholders is related to the complexity of managing a vast array of perspectives and representing everyone's interests equally. This is confirmed in a study comparing the Athena SWAN award scheme with other gender equality initiatives in Europe, which found that interventions are complex social interventions in a complex system
[29]. A multitude of contextual variables relate in complex, non-linear ways and must constantly adapt to moving targets and new conditions. Studies that include sex and gender considerations and other intersecting factors may be complex but are a more inclusive and therefore faithful representation (or measure) of reality. Yet, the irony is that the knowledge that KT studies are expanding and disseminating is ultimately produced by the scientific method, which is often more about excluding rather than including. It is unsurprising then that the scientific community may not always welcome this complexity because it calls into question the paradigm of the scientific method itself [30]. Further research is needed on the obstacles that prevent investigators from taking account of gender and sex, on papers that have influenced investigators in a positive way and how this happened, and on best forums for promoting discussion of these issues.

The paper is subject to several limitations. The meeting was part of the CIHR Impact of Gender on KT Interventions grant and, as such, it was not initially planned for data collection and analysis; in addition, the discourse of team members present at the discussion may not have been representative of the key researchers in each project. Second, the meeting was held in a high-income country with a resource-intensive health system; thus, the findings may not represent all the challenges in this respect, although a few participants were from LMICs and one of the teams had a project in a LMIC (Table 1). While a study on child development that covered 41 LMICs found that few major gender differences emerged [31], supporting a gender similarities view, the integration of sex and gender into KT interventions would vary widely depending on institutional and cultural norms. Finally, there were no patient participants at the meeting, although some teams included patient partners.

\section{Conclusions}

These findings provide a sampling of the challenges and solutions found in implementing KT projects that consider sex and gender. They have implications for every stage of the KT process, from identifying the knowledge to be translated to disseminating it in an equitable way. Integrating sex and gender in $\mathrm{KT}$ is a new field and these findings will illuminate the path ahead.

\section{Supplementary information}

Supplementary information accompanies this paper at https://doi.org/10. 1186/s12961-020-00625-6.

Additional file 1. Agenda of the impact of gender on KT interventions team grant recipient meeting.

Additional file 2. List of team representatives. 


\section{Abbreviations}

CIHR: Canadian Institutes of Health Research; CPD: continuing professional development; IGH: Institute of Gender and Health; KT: knowledge translation; LMICs: low- and middle-income countries; TBI: traumatic brain injury; TDF: Theoretical Domains Framework

\section{Acknowledgements}

We thank Louisa Blair, editor at Vitam - Centre de recherche en santé durable, Quebec, Canada, for her support.

We also thank the Gender Impact on Knowledge Translation (GIKT) Group: Lionel Adisso, Department of Social and Medicine, Université Laval; Sylvain Boet, Department of Anesthesiology and Pain Medicine \& Department of Innovation in Medical Education, University of Ottawa; Andreea C. Brabete, Department of Personality, Centre of Excellence for Women's Health, University of British Columbia; Angela Colantonio, Rehabilitation Sciences Institute, University of Toronto; Cole Etherington, Centre for Practice Changing Research, Ottawa Hospital Research Institute; Amédé Gogovor, Department of Family Medicine and Emergency Medicine, Université Laval; Lorraine Greaves, Centre of Excellence for Women's Health, University of British Columbia; Marie Laberge, School of Rehabilitation, Université de Montréal; France Légaré, Department of Family Medicine and Emergency Medicine, Université Laval; Karen Messing, Biological Sciences, Université du Québec à Montréal; Tatyana Mollayeva, Rehabilitation Sciences Institute, University of Toronto; Sylvie-Marianne Rhugenda, Faculty of Social Sciences, Université Laval; Kathryn Sibley, Department of Community Health Sciences, University of Manitoba; Cora Siebert, Institute of Gender and Health, Canadian Institutes of Health Research; Sharon Straus, St. Michael's Hospital, University of Toronto; Dominique Tanguay, Faculty of Social Sciences, Université Laval; Cara Tannenbaum, Institute of Gender and Health, Canadian Institutes of Health Research; and Cathy Vaillancourt, Institut national de recherche scientifique, Centre Armand-Frappier Santé Biotechnologie.

\section{Authors' contributions}

AG, TM, CE, AC and FL conceptualised the paper. All authors contributed to data generation. AG and TM analysed and synthesised the data. All the authors contributed to the preparation and critical review of the manuscript. All listed authors and other members of the GIKT Group read and approved the final manuscript.

\section{Funding}

The meeting was supported by the CIHR IGH Impact of Gender on Knowledge Translation Interventions grant IGK-153466 from Team 1. AG received a postdoctoral fellowship from the Fonds de recherche du QuébecSanté (FRQS) and the Quebec SPOR-SUPPORT Unit. FL holds a Tier 1 Canada Research Chair in Shared Decision Making and Knowledge Translation.

\section{Availability of data and materials}

The text files used and/or analysed during the current study are available from the corresponding author on reasonable request.

\section{Ethics approval and consent to participate}

Verbal consent was obtained from the participants regarding further use of the output of the meeting. The Centre intégré universitaire de santé et de services sociaux de la Capitale-Nationale Ethics Board certified that ethical approval was not required.

\section{Consent for publication}

Not applicable.

\section{Competing interests}

The authors declare that they have no competing interests.

\section{Author details}

'Vitam - Centre de recherche en santé durable, Pavillon Landry-Poulin, 2525, Chemin de la Canardière, Québec G1J 0A4, Canada. ${ }^{2}$ Tier 1 Canada Research Chair in Shared Decision Making and Knowledge Translation, Université Laval, Quebec, Canada. ${ }^{3}$ Health and Social Services Systems, Knowledge Translation and Implementation Component of the Quebec SPOR-SUPPORT Unit, Quebec, Canada. ${ }^{4}$ Department of Family Medicine and Emergency Medicine, Université Laval, Quebec, Canada. ${ }^{5}$ Rehabilitation Sciences Institute, Faculty of Medicine, University of Toronto, 160-500 University Avenue,
Toronto M5G 1V7, Canada. ${ }^{6}$ Toronto Rehabilitation Institute-University Health Network, University of Toronto, 160-500 University Avenue, Toronto M5G 1V7, Canada. ${ }^{7}$ Clinical Epidemiology Program, Ottawa Hospital Research Institute, 501 Smyth Road, Rm L1287, Ottawa, ON K1H 8L6, Canada.

Received: 2 March 2020 Accepted: 30 August 2020

Published online: 23 September 2020

\section{References}

1. Canadian Institutes of Health Research (CIHR). Knowledge Translation at CIHR. http://www.cihr-irsc.gc.ca/e/29418.html. Accessed 21 Dec 2018.

2. Sudsawad P. Knowledge translation: Introduction to models, strategies, and measures. Austin, TX: Southwest Educational Development Laboratory, National Center for the Dissemination of Disability Research; 2007.

3. Tricco AC, Ashoor HM, Cardoso R, et al. Sustainability of knowledge translation interventions in healthcare decision-making: a scoping review. Implement Sci. 2016;11:55.

4. Tannenbaum C, Greaves L, Graham ID. Why sex and gender matter in implementation research. BMC Med Res Methodol. 2016;16:145.

5. Greaves L. Why put Gender and Sex into Health Research? In: Designing and conducting gender, sex, and health research. Thousand Oaks, CA: Sage Publications, Inc.; 2012

6. Canadian Institutes of Health Research (CIHR). Sex, Gender and Knowledge Translation. http://www.cihr-irsc.gc.ca/e/49933.html. Accessed 21 Dec 2018.

7. Canadian Institutes of Health Research (ClHR). Gender, sex, and health research guide: a tool for CIHR applicants. http://www.cihr-irsc.gc.ca/e/5 0836.html. Accessed 21 Dec 2018

8. Hankivsky O, Cormier R. Intersectionality: Moving Women's Health Research and Policy Forward. Women's Health Research Network: Vancouver; 2009.

9. O'Neill J, Tabish H, Welch V, Petticrew M, Pottie K, Clarke M, et al. Applying an equity lens to interventions: using PROGRESS ensures consideration of socially stratifying factors to illuminate inequities in health. J Clin Epidemiol. 2014;67:56-64

10. Springer KW, Mager Stellman J, Jordan-Young RM. Beyond a catalogue of differences: a theoretical frame and good practice guidelines for researching sex/gender in human health. Soc Sci Med. 2012;74(11):1817-24.

11. Rosser SV, Barnard S, Carnes M, Munir F. Athena SWAN and ADVANCE: effectiveness and lessons learned. Lancet. 2019;393(10171):604-8.

12. Crenshaw K. Demarginalizing the Intersection of Race and Sex: A Black Feminist Critique of Antidiscrimination Doctrine, Feminist Theory and Antiracist Politics. Chicago: University of Chicago Legal Forum; 1989.

13. Hill Collins P, Bilge S. Intersectionality. Chichester: Wiley; 2016.

14. Etherington N, Rodrigues IB, Giangregorio L, Graham ID, Hoens AM, Kasperavicius D, et al. Applying an intersectionality lens to the theoretical domains framework: a tool for thinking about how intersecting social identities and structures of power influence behaviour. BMC Med Res Methodol. 2020;20:169

15. Mena E, Bolte G. Intersectionality-based quantitative health research and sex/gender sensitivity: a scoping review. Int J Equity Health. 2019;18:199.

16. Mollayeva T, Amodio V, Mollayeva $S$, et al. A gender-transformative approach to improve outcomes and equity among persons with traumatic brain injury. BMJ Open. 2019;9(5):e024674.

17. Rottach E, Hardee K, Jolivet R, Kiesel R. Integrating Gender into the Scale-Up of Family Planning and Maternal, Neonatal, and Child Health Programs. Washington, DC: Futures Group, Health Policy Project; 2012.

18. World Health Organization. Gender, Women and Primary Health Care Renewal. Discussion Paper. Geneva: WHO; 2010.

19. World Health Organization. Mainstreaming gender equity in health: The need to move forward. Madrid Statement. Denmark: WHO EURO; 2002.

20. World Health Organization Commission on Social Determinants of Health. Closing the gap in a generation: health equity through action on the social determinants of health. Final Report of the Commission on Social Determinants of Health. Geneva: WHO; 2008.

21. Braun V, Clarke V. Using thematic analysis in psychology. Qual Res Psychol. 2006:3(2):77-101.

22. Johnson JL, Greaves L, Repta R. Better science with sex and gender: Facilitating the use of a sex and gender-based analysis in health research. Int J Equity Health. 2009:8:14.

23. Ratner P, Sawatzky R. Approaches to the measurement of gender. In: Oliffe $J$, Greaves L, editors. Designing and conducting gender, sex, and health research. Thousand Oaks, CA: Sage Publications, Inc; 2012. p. 65-85. 
24. Day S, Mason R, Tannenbaum C, Rochon PA. Essential metrics for assessing sex \& gender integration in health research proposals involving human participants. PLoS One. 2017;12(8):e0182812.

25. Day S, Mason R, Lagosky S, Rochon PA. Integrating and evaluating sex and gender in health research. Health Res Policy Syst. 2016;14:75.

26. Sugimoto $C R$, Ahn $Y-Y$, Smith $E$, Macaluso B, Larivière $V$. Factors affecting sex-related reporting in medical research: a cross-disciplinary bibliometric analysis. Lancet. 2019;393(10171):550-9.

27. TDR Intersectional Gender Research Strategy: building the science of solutions for all. Geneva: WHO; 2020.

28. Hankivsky O, Springer KW, Hunting G. Beyond sex and gender difference in funding and reporting of health research. Res Integr Peer Rev. 2018;3:6.

29. Kalpazidou Schmidt E, Ovseiko PV, Henderson LR, Kiparoglou V. Understanding the Athena SWAN award scheme for gender equality as a complex social intervention in a complex system: analysis of Silver award action plans in a comparative European perspective. Health Res Policy Syst. 2020;18:19.

30. Greenhalgh T, Papoutsi C. Studying complexity in health services research: desperately seeking an overdue paradigm shift. BMC Med. 2018;16:95.

31. Bornstein MH, Putnick DL, Bradley RH, Deater-Deckard K, Lansford JE. Gender in low- and middle-income countries: introduction. Monogr Soc Res Child Dev. 2016:81(1):7-23.

32. Laberge M, Blanchette-Luong V, Blanchard A, Sultan-Taieb H, Riel J, Lederer $V$, et al. Impacts of considering sex and gender during intervention studies in occupational health: researchers' perspectives. Appl Ergon. 2019;82: 102960.

\section{Publisher's Note}

Springer Nature remains neutral with regard to jurisdictional claims in published maps and institutional affiliations.

Ready to submit your research? Choose BMC and benefit from:

- fast, convenient online submission

- thorough peer review by experienced researchers in your field

- rapid publication on acceptance

- support for research data, including large and complex data types

- gold Open Access which fosters wider collaboration and increased citations

- maximum visibility for your research: over $100 \mathrm{M}$ website views per year

At BMC, research is always in progress.

Learn more biomedcentral.com/submissions 